\title{
FUNCTIONS OF EXPONENTIAL TYPE IN AN ANGLE AND SINGULARITIES OF TAYLOR SERIES
}

\author{
BY \\ SHMUEL AGMON
}

1. Introduction. A function $f(z)$, analytic in the angle $|\arg z| \leqq \alpha$, is said to be of exponential type in the angle if there exist two constants $a$ and $A$ such that

$$
|f(z)| \leqq A e^{a|z|} \quad(|\arg z| \leqq \alpha) .
$$

It was shown by Cartwright [10] $\left(^{1}\right)$ that if $f(z)$ satisfies (1.1) and a condition which is essentially

$$
\limsup _{r=\infty} \frac{\log \left|f\left(r e^{ \pm i \alpha}\right)\right|}{r} \leqq k \sin \alpha \quad(0 \leqq k<\pi),
$$

and if $f(z)$ is bounded on the sequence of points $z=1,2, \cdots$, then $f(z)$ is bounded on the entire positive axis. (See also Macintyre [21].)

It was shown by Boas (for entire functions) [6] and more generally by Duffin and Schaeffer [13] (for $\alpha=\pi / 2$ ) that Cartwright's theorem remains true if the sequence of integers $n=1,2, \cdots$, where $f(z)$ is bounded, is replaced by a more general sequence of complex numbers $\left\{\lambda_{n}\right\}$. The condition imposed by Duffin and Schaeffer on the $\lambda_{n}$ 's, and which will be retained by us through most of this paper, is

$$
\left|\lambda_{n}-\lambda_{m}\right| \geqq h>0(n \neq m) \text { and }\left|\lambda_{n}-n\right| \leqq H
$$

where $h$ and $H$ are two constants.

We propose to remove here the condition of boundedness of $f\left(\lambda_{n}\right)$ and to obtain similar results concerning the growth of the function from its growth on a sequence of points, under the much more general and "natural" restriction

$$
\limsup _{n=\infty} \frac{\log \left|f\left(\lambda_{n}\right)\right|}{\left|\lambda_{n}\right|} \leqq 0 .
$$

We shall show that if $f(z)$ satisfies (1.1) and (1.2), and if $\left\{\lambda_{n}\right\}$ is a sequence of complex numbers for which (1.3) and (1.4) are satisfied, then there exists a "regular" majorant $\left\{q_{n}\right\}$ of $\left\{\left|f\left(\lambda_{n}\right)\right|\right\}$ such that for $k^{\prime}>k$ and $z=x+i y$ in the angle, we have

$$
|f(x+i y)| \leqq C q_{[x]} e^{k^{\prime}|y|}
$$

Received by the editors December 15, 1949 and, in revised form, August 4, 1950 .

(1) Numbers in brackets refer to the bibliography at the end of the paper. 
where $C=C\left(h, H, k^{\prime} ; f\right)$ is a constant $\left({ }^{2}\right)$.

Now, if the sequence $\left\{\left|f\left(\lambda_{n}\right)\right|\right\}$ is regular enough, $q_{[x]}$ in (1.5) can be replaced by $\left|f\left(\lambda_{[x]}\right)\right|$. In the general case, if (1.4) is satisfied with the sign of equality, there exists a touching majorant $\left\{q_{n}\right\}$ for which (1.5) holds; where by a touching majorant we mean a sequence $\left\{q_{n}\right\}$, such that

$$
q_{n} \geqq\left|f\left(\lambda_{n}\right)\right|
$$$$
(n=1,2, \cdots) \text { while } q_{n_{k}}=\left|f\left(\lambda_{n_{k}}\right)\right|
$$

for an infinite subsequence $\left\{n_{k}\right\}$.

We would like to make two remarks. The first concerns the three conditions (1.1), (1.2), and (1.4), which we say are equivalent to (1.1) and

$$
\left.\limsup _{r=\infty} \frac{\log \left|f\left(r e^{i \theta}\right)\right|}{r} \leqq k|\sin \theta| \quad \text { (for }|\theta| \leqq \alpha\right),
$$

where $k$ is such that $0 \leqq k<\pi$, if the sequence $\left\{\lambda_{n}\right\}$ verifies (1.3) or, more generally if $\lim \left(\lambda_{n} / n\right)=1$. Indeed, it is obvious that (1.7) and (1.1) imply (1.2) and (1.4). To show the converse we apply a result of $V$. Bernstein [5], in a generalized form given by Pfluger [24], by which the last mentioned three conditions, with $\lim \left(\lambda_{n} / n\right)=1$, imply $\lim \sup _{x=\infty}[(\log |f(x)|) / x] \leqq 0$. But, it is well known (by the method of Phragmén and Lindelöf) that the last relation, when combined with (1.1) and (1.2), yields (1.7).

In the second remark we want to point out that there exists an almost trivial method of getting some results in the general case which will depend on the theorem of Duffin and Schaeffer for the special case when $f\left(\lambda_{n}\right)$ is bounded. Thus, we can construct a function $\eta(z)$ analytic in the angle, satisfying there $\eta(z) / z \rightarrow 0$, and such that $\Re\left[\eta\left(\lambda_{n}\right)\right] \geqq \log \left|f\left(\lambda_{n}\right)\right|$. Applying the theorem of Duffin and Schaeffer (slightly generalized) to the function $f(z) e^{-\eta(z)}$, we shall obtain (1.5) with $q_{n}=e^{\Re\left[\eta\left(\lambda_{n}\right)\right]}$. The point is, however, that this method gives results which are not precise enough, for, in almost all the applications of our theorem, it is essential to have touching majorant sequences $\left\{q_{n}\right\}$ for which the theorem is applicable. But, it seems to us that it is impossible (in the general case) to construct an analytic function $\eta(z)$ with the above properties and such that $\left\{e^{\Re\left[\eta\left(\lambda_{n}\right)\right]}\right\}$ will be a touching majorant of $\left\{\left|f\left(\lambda_{n}\right)\right|\right\}$.

Finally we mention that a large part of this paper is devoted to applications of the main theorem described above to problems of singularities of Taylor series. Thus, in $\$ 4$ we prove a general gap theorem; in $\$ 5$ we generalize a theorem of Szegö; and in $\$ 6$ we prove a weak converse of a theorem of Fabry.

2. Some lemmas. We shall use the following known inequality, announced as a lemma, which is an immediate consequence of the PoissonNevanlinna formula for the half-circle. (See F. and R. Nevanlinna [22]. For a proof see also Levinson [19, p. 245].)

(2) It can be easily shown, though we shall not prove it here, that for $x$ large enough $C$ can be replaced by $C_{0}=C_{0}\left(h, H, k^{\prime}\right)$. 
Lemma 2a. Let $g(w)$ be analytic in the half-circle $|w| \leqq R, v \geqq 0$, where $w=u+i v=\rho e^{i \psi}$. Then, for $w$ inside the circle,

$$
\frac{\log |g(u+i v)|}{v} \leqq \frac{1}{\pi} \int_{-R}^{R} \log |g(t)| N(w, R, t) d t
$$

$$
+\frac{1}{\pi} \int_{0}^{\pi} \log \left|g\left(R e^{i \phi}\right)\right| P(w, R, \phi) d \phi,
$$

where both $N(w, R, t)$ and $P(w, R, \phi)$ are positive kernels, given explicitly by

$$
N(w, R, t)=\frac{\left(R^{2}-\rho^{2}\right)\left(R^{2}-t^{2}\right)}{\left(t^{2}-2 \rho t \cos \psi+\rho^{2}\right)\left(R^{4}-2 \rho t R^{2} \cos \psi+\rho^{2} t^{2}\right)}
$$

$$
=\frac{1}{t^{2}-2 \rho t \cos \psi+\rho^{2}}-\frac{R^{2}}{R^{4}-2 \rho t R^{2} \cos \psi+\rho^{2} t^{2}},
$$

and

$$
P(w, R, \phi)=\frac{2 R\left(R^{2}-\rho^{2}\right) \sin \phi}{\left|R^{2} e^{i 2 \phi}-2 \rho R \cos \psi e^{i \phi}+\rho^{2}\right|^{2}} .
$$

Moreover, if $g(w) \neq 0$ in the half-circle, then (2.1) becomes equality. Taking, in particular, $g(w)=e^{i w}$, we have identically,

$$
1 \equiv \frac{R}{\pi} \int_{0}^{\pi} \sin \phi P(w, R, \phi) d \phi .
$$

The following lemma can be considered as a weak form of the main theorem. We shall suppose in it that the value of the function $f(z)$ of exponential type is known on the whole positive axis and not only on a sequence of points.

LEMMA 2b. Let $f(z)$ be an analytic function of exponential type in the angle $|\arg z| \leqq \alpha<\pi / 2$, satisfying (1.7) with $k$ non-negative (but not necessarily less than $\pi)$. Suppose that on the positive axis

$$
|f(x)| \leqq p(x),
$$

where $p(x)$ is a positive function with the following properties:

$$
\log p(x)=\int_{0}^{x} \omega(t) d t+\text { const., }
$$

$\omega(x)$ being Riemann-integrable in any finite interval such that

$$
\lim _{x=\infty} \omega(x)=0,
$$


and

$$
\omega\left(x^{\prime}\right)-\omega(x) \leqq \frac{A}{x} \quad \text { for } 0<x<\infty, 0 \leqq x^{\prime}-x \leqq 1,
$$

and some non-negative constant $A$.

Then if $k^{\prime}>k$ and $\eta>1$, there exists a positive number $x_{0}$ such that

$$
|f(x+i y)|<\eta p(x) e^{k^{\prime}|y|},
$$

for $z$ satisfying $|\arg z| \leqq \alpha$ and $x \geqq x_{0}$.

(We remark that (i), (ii), and (iii) will be satisfied, in particular, if $\log p(x)$ is a concave function of $x$, and $\log p(x)=o(x)$. It will also be satisfied if $\log p(x)$ is twice differentiable with $\lim _{x=\infty}(\log p(x))^{\prime}=0$ and $(\log p(x))^{\prime \prime}$ $\leqq A / x$.)

Proof. In what follows $o(1)$ will denote a positive function of $x$ tending to zero when $x \rightarrow \infty$. It is well known that (1.7) holds essentially uniformly, so

$$
\log \left|f\left(r e^{i \theta}\right)\right| \leqq k|\sin \theta| r+o(1) \cdot r=k|y|+o(1) \cdot r \quad(|\theta| \leqq \alpha) .
$$

It is also easily verified (taking account of (2.6) and the fact that $|\log p(x)| / x$ $=o(1)$ ) that the inequality to be proved, (2.5), holds trivially outside any angle $|\arg z| \leqq \alpha^{\prime}<\alpha$. Let us choose a number $\lambda$ such that

$$
0<\lambda<\min \left(\frac{1}{2}, \frac{\pi}{4 A} \log \eta, \frac{\pi}{4 A}\left(k^{\prime}-k\right), \sin \alpha\right)
$$

where $A$ is the constant satisfying (iii), and let $\alpha^{\prime}$ be a positive number such that $\tan \alpha^{\prime}<\lambda$. Obviously $\alpha^{\prime}<\alpha$ and it is clear from the preceding that it is enough to prove (2.5) for $z$ satisfying $|\arg z|<\alpha^{\prime}$. Let $z=x+i y$ be any such point and suppose to begin with that $y$ is positive. Put

$$
g(w)=g_{x}(w)=\frac{f(x+w)}{p(x)} .
$$

The function $g(w)$ is holomorphic in the half-circle $|w| \leqq \lambda x, v \geqq 0(w=u+i v)$. Hence, applying Lemma $2 \mathrm{a}$ to this function and the point $w=i y$ (which is inside the half-circle on account of our previous suppositions) we can write

$$
\begin{aligned}
& \frac{\log |f(x+i y)|-\log p(x)}{\leqq} \frac{1}{\pi} \int_{-\lambda x}^{\lambda x}\{\log |f(x+t)|-\log p(x)\} N(i y, \lambda x, t) d t \\
& +\frac{1}{\pi} \int_{0}^{\pi}\left\{\log \left|f\left(x+\lambda x e^{i \phi}\right)\right|-\log p(x)\right\} P(i y, \lambda x, \phi) d \phi=I_{1}+I_{2} .
\end{aligned}
$$

The kernel $N$ being positive and given by (2.2) we find for $I_{1}$, using (2.4), 


$$
\begin{aligned}
I_{1} \leqq & \frac{1}{\pi} \int_{-\lambda x}^{\lambda x}\{\log p(x+t)-\log p(x)\} N(i y, \lambda x, t) d t \\
= & \frac{1}{\pi} \int_{0}^{\lambda x}\{\log p(x+t)+\log p(x-t)-2 \log p(x)\} \frac{d t}{t^{2}+y^{2}} \\
& -\frac{1}{\pi} \int_{-\lambda x}^{\lambda x}\{\log p(x+t)-\log p(x)\} \frac{\lambda^{2} x^{2}}{\lambda^{4} x^{4}+y^{2} t^{2}}=J_{1}-J_{2} .
\end{aligned}
$$

Now, it is easily seen from the properties of $p(x)$ that for positive $x$ and $0<t \leqq x / 2$,

(2.10) $\log p(x+t)+\log p(x-t)-2 \log p(x)<\frac{4 A}{x} t(t+1)$.

(Indeed, we have $\log p(x+t)+\log p(x-t)-2 \log p(x)=\int_{0}^{t}[\omega(x+u)$ $-\omega(x-u)] d u$, and, from (iii), $\omega(x+u)-\omega(x-u)<4(u+1) A / x$ for $0 \leqq u$ $\leqq x / 2$, so that (2.10) follows from the last two relations.) Using (2.10) in the expression for $J_{1}$, we get (using the inequality $\log (1+b)<2(b)^{1 / 2}(b>0)$ ),

$$
\begin{aligned}
J_{1} & <\frac{4 A}{\pi x} \int_{0}^{\lambda x}\left(1+\frac{t}{t^{2}+y^{2}}\right) d t=\frac{4 A \lambda}{\pi}+\frac{2 A}{\pi x} \log \left(1+\frac{\lambda^{2} x^{2}}{y^{2}}\right) \\
& <\frac{4 A \lambda}{\pi}+\frac{4 A \lambda}{\pi y} .
\end{aligned}
$$

For $J_{2}$ we find, using properties (i) and (ii) of $p(x)$

$$
\left|J_{2}\right| \leqq \frac{1}{\pi} \int_{-\lambda x}^{\lambda x}|\log p(x+t)-\log p(x)| \frac{\lambda^{2} x^{2}}{\lambda^{4} x^{4}+y^{2} t^{2}} d t
$$

$$
<\left(\max _{|l| \leqq \lambda x}|\log p(x+t)|\right) \frac{4}{\pi \lambda x}=o(1) .
$$

Combining (2.9), (2.11), and $\left(2.11^{\prime}\right)$ we obtain,

$$
I_{1} \leqq J_{1}+\left|J_{2}\right| \leqq \frac{4 A \lambda}{\pi}\left(1+\frac{1}{y}\right)+o(1) .
$$

Now, if $x+\lambda x e^{i \phi}=r e^{i \theta}$, then $\lambda x \sin \phi=r \sin \theta$, so that (2.6) can be rewritten as

$$
\log \left|f\left(x+\lambda x e^{i \phi}\right)\right| \leqq k \lambda x \sin \phi+o(1) \cdot x
$$

Also from (2.2'),

$$
0 \leqq P(i y, \lambda x, \phi) \leqq \frac{C}{x}
$$


where $C=C\left(\lambda, \alpha^{\prime}\right)$ is a constant. Using (2.12), $\left(2.12^{\prime}\right)$, and the identity (2.3), remembering that $|\log p(x)| / x=o(1)$, we get

$$
\begin{aligned}
I_{2} & =\frac{1}{\pi} \int_{0}^{\pi}\left\{\log \left|f\left(x+\lambda x e^{i \phi}\right)\right|-\log p(x)\right\} P(i y, \lambda x, \phi) d \phi \\
& \leqq \frac{k}{\pi} \int_{0}^{\pi} \lambda x \sin \phi P(i y, \lambda x, \phi) d \phi+o(1)=k+o(1) .
\end{aligned}
$$

Finally, combining $(2.8),\left(2.11^{\prime \prime}\right),(2.13)$, and using (2.7), we find,

$$
\begin{aligned}
\log |f(x+i y)|-\log p(x) & \leqq\left(k+\frac{4 A \lambda}{\pi}+o(1)\right) \cdot y+\frac{4 A \lambda}{\pi} \\
& \leqq k^{\prime} y+\log \eta \quad \text { for } x \geqq x_{0},
\end{aligned}
$$

which is the desired inequality (2.5) for $y>0$. For negative $y$ ( $y=0$ is trivial) we have only to apply the proved result to $f(\bar{z})$.

3. The main theorem. We shall begin by discussing in some detail the properties of the smallest concave majorant, defined for a certain class of real functions, which turn out to be of fundamental importance in our proof of the main theorem.

Let $\omega(x)$ be a real and continuous function for $0 \leqq x<\infty$, such that $\lim \sup (\omega(x) / x)=0$. Then, there exists a unique function $C(x)$ having the following three properties:

(i) $C(x)$ is a majorant of $\omega(x): C(x) \geqq \omega(x)$.

(ii) $C(x)$ is concave: $C(x+h)+C(x-h)-2 C(x) \leqq 0(h>0, x-h \geqq 0)$.

(iii) $C(x)$ is the smallest function having properties (i) and (ii).

The function $C(x)$ is called the smallest concave majorant (or envelope) of $\omega(x)$. Indeed, we can define $C(x)(0 \leqq x<\infty)$ as the smallest number $\gamma$ such that there exists a straight line passing through $(x, \gamma)$ above which there are no points of the curve $y=\omega(x)$. It is easily proved that $C(x)$ so defined is concave. That it is a majorant is obvious; and that it is the smallest concave majorant follows immediately from the construction.

Now, from the condition of growth imposed on $\omega(x)$, it follows in an easy way that $C(x) / x \rightarrow 0$, and this combined with concavity shows that the slope of $C(x)$ is always non-negative. Hence, $C(x)$ is nondecreasing, and we also have $\lim _{x=\infty} C(x)=$ upper bound ${ }_{0 \leqq x<\infty} \omega(x)$. The interesting case, which will also concern us, is when $\omega(x)$ is not bounded from above, so that $C(x) \uparrow \infty$. In this case there exists an infinite sequence of positive numbers $\left\{\xi_{n}\right\}$ tending to infinity (a principal sequence), such that

$$
C\left(\xi_{n}\right)=\omega\left(\xi_{n}\right) \quad(n=1,2, \cdots) .
$$

Indeed, let $\left\{\xi_{n}\right\}$ be a sequence of positive numbers tending to infinity, such that $y=C(x)$ is not linear in any small interval with $\left\{\xi_{n}\right\}$ as center. Such a 
sequence exists, for otherwise $y=C(x)$ would be a straight line with a nonnegative slope for $x$ large enough. However, the slope of the line cannot be positive, since $C(x) / x \rightarrow 0$; and it cannot be zero because $C(x)$ is not bounded. Now, the last defined sequence $\left\{\xi_{n}\right\}$ satisfies (3.1). For, if $C\left(\xi_{n}\right)>\omega\left(\xi_{n}\right)$, there exists $\delta>0$ sufficiently small such that $\omega(x) \leqq l(x)$ for $\xi_{n}-\delta \leqq x \leqq \xi_{n}+\delta$, where $l(x)$ is the linear function joining $\left(\xi_{n}-\delta, C\left(\xi_{n}-\delta\right)\right)$ and $\left(\xi_{n}+\delta, C\left(\xi_{n}+\delta\right)\right)$. Thus, we shall get another concave majorant $C^{*}(x)$ by putting $C^{*}(x)=l(x)$ in the interval $\left(\xi_{n}-\delta, \xi_{n}+\delta\right)$ and $C^{*}(x)=C(x)$ elsewhere, Or, $C^{*}(x) \leqq C(x)$ $(x \geqq 0)$ and $C^{*}(x)<C(x)$ for some point in $\left(\xi_{n}-\delta, \xi_{n}+\delta\right)(C(x)$ is not linear there). This contradicts the definition of $C(x)$ and establishes (3.1).

We pass now to the statement and proof of the main theorem( $\left.{ }^{3}\right)$.

THEOREM 3a. Let $f(z)$ be an analytic function of exponential type in the angle $|\arg z| \leqq \alpha<\pi / 2$, such that

$$
\limsup _{x=\infty} \frac{\log \left|f\left(r e^{i \theta}\right)\right|}{r} \leqq k|\sin \theta| \quad \text { for }|\theta| \leqq \alpha,
$$

and where $0 \leqq k<\pi$. Let $\left\{\lambda_{n}\right\}$ be a sequence of complex numbers which satisfies

$$
\left|\lambda_{n}-\lambda_{m}\right| \geqq h>0(n \neq m) \quad \text { and } \quad\left|\lambda_{n}-n\right| \leqq H \quad(n=1,2, \cdots),
$$

$h$ and $H$ being two positive constants. Suppose, furthermore, that

$$
\left|f\left(\lambda_{n}\right)\right| \leqq q_{n} \quad(n=1,2, \cdots),
$$

where $\left\{q_{n}\right\}$ is a sequence of positive numbers satisfying the following conditions of regularity

$$
\lim q_{n} / q_{n+1}=1
$$

$$
(n \rightarrow \infty),
$$

and

$$
\log q_{n+1}+\log q_{n-1}-2 \log q_{n} \leqq B / n \quad(n=2,3, \cdots)
$$

for some constant B. Define $q(x)$ to be continuous, $q(n)=q_{n}$, and $\log q(x)$ linear for $n \leqq x \leqq n+1$. Then, if $k^{\prime}>k$, there exists a constant $C$ such that, for $|\arg z|$ $\leqq \alpha$,

$$
|f(x+i y)| \leqq C q(x) e^{k^{\prime}|\nu|}
$$

Proof. Put $\left(^{4}\right)$

$$
\omega(x)=\log ^{+}\left(\frac{|f(x)|}{q(x)}\right)
$$$$
(x \geqq 0) \text {, }
$$

so that $\omega(x)$ is continuous, and lim $\sup (\omega(x) / x)=0$. It is easily seen that $q(x)$

(3) This theorem was announced by us in a somewhat less general form (as concerns the sequence $\left.\left\{q_{n}\right\}\right)$ in $[2]$.

(4) If $A$ is a real number we write, as usual, $A^{+}$for $(A+|A|) / 2$. 
satisfies properties (i), (ii), and (iii) of Lemma $2 b$. Thus, if we use this lemma, it is enough to prove that $\omega(x)$ is bounded in order to obtain our result. Suppose now the contrary, that the theorem is false and that we have

$$
\lim _{x=\infty} \sup \omega(x)=\infty \text {. }
$$

Let $C(x)$ be the smallest concave majorant of $\omega(x)$. It follows from what we have shown in the beginning of this section that $C(x) / x \rightarrow 0$, and $C(x) \uparrow \infty$. Moreover, there exists a sequence $\left\{\xi_{n}\right\}$ of positive numbers tending to infinity, such that

$$
\omega\left(\xi_{n}\right)=C\left(\xi_{n}\right)
$$$$
(n=1,2, \cdots) \text {. }
$$

Let us put $p(x)=q(x) e^{C(x)}$. It follows from (3.6), the definition of $C(x)$, and (3.7), that

$$
|f(x)| \leqq p(x) \quad(0 \leqq x<\infty) \text { and }\left|f\left(\xi_{n}\right)\right|=p\left(\xi_{n}\right) \quad(n=1,2, \cdots) .
$$

Furthermore, it is easy to verify that both functions $q(x)$ and exp $[C(x)]$, and consequently their product $p(x)$, satisfy hypotheses (i), (ii), and (iii) of Lemma 2b. Applying this lemma we find that if $\eta>1$ and $k^{\prime}>k$, there exists $x_{0}$ such that for $|\arg z| \leqq \alpha, x \geqq x_{0}$,

$$
|f(x+i y)| \leqq \eta p(x) e^{k^{\prime}|y|} .
$$

Let us consider now the family of functions $\left\{F_{n}(z)\right\}$ defined by

$$
F_{n}(z)=\frac{f\left(\xi_{n}+z\right)}{f\left(\xi_{n}\right)} \quad(n=1,2, \cdots),
$$

which are analytic inside any circle $|z| \leqq R$ for $n \geqq n_{0}=n_{0}(R)$, and satisfy there, on account of (3.9) and (3.8),

$$
\left|F_{n}(x+i y)\right| \leqq \eta \frac{p\left(\xi_{n}+x\right)}{p\left(\xi_{n}\right)} e^{k^{\prime}|y|}
$$

Or, we have uniformly for $x$ in any fixed interval $|x| \leqq R$,

$$
\lim _{n=\infty}\left(p\left(\xi_{n}+x\right) / p\left(\xi_{n}\right)\right)=1,
$$

so that the family $\left\{F_{n}(z)\right\}$ is bounded in any finite circle and hence is normal. Let $G(z)$ be any limit function of the family:

$$
G(z)=\lim _{\nu=\infty} F_{n_{\nu}}(z)
$$

It follows from the above remarks that $G(z)$ is an entire function not identically zero, $G(0)=1$, which, on account of (3.11) and (3.12) (remembering that the only restrictions on $\eta$ and $k^{\prime}$ are $\eta>1$ and $k^{\prime}>k$ ) satisfies 


$$
|G(z)| \leqq e^{k|y|} .
$$

We are now going to get a contradiction by showing that $G(z)$ has too many zeros to be consistent with its growth. Let $n(r)$ denote the number of zeros of $G(z)$ inside the circle $|z| \leqq r$. We claim that

$$
n(r) \geqq 2 r-O(1)
$$$$
(r \rightarrow \infty) .
$$

For, let $m_{\nu}=\left[\xi_{n_{\nu}}\right]([x]$ being as usual the largest integer not exceeding $x$, and $\left\{n_{\nu}\right\}$ the subsequence of $\left.(3.13)\right)$ and put

$$
\mu_{j}^{(\nu)}=\lambda_{m_{\nu}+j}-\xi_{n_{\nu}}, \quad \text { for } j \geqq-m_{\nu} .
$$

It follows from (3.3) that

$$
\begin{aligned}
\left|\mu_{j_{2}}^{(\nu)}-\mu_{j_{1}}^{(\nu)}\right| & \geqq h>0 \quad\left(j_{2}>j_{1} \geqq-m_{\nu}\right) \quad \text { and } \\
\left|\mu_{j}^{(\nu)}-j\right| & \leqq H+1 \quad\left(j \geqq-m_{\nu}\right),
\end{aligned}
$$

and it is now obvious from (3.17) that by a diagonal process we can choose a subsequence $\left\{\nu_{i}\right\}$, such that

$$
\lim _{i=\infty} \mu_{i}^{\left(\nu_{1}\right)}=\mu_{i} \quad(j=0, \pm 1, \cdots) .
$$

Now, all the $\mu_{j}$ 's are zeros of $G(z)$. Indeed, using (3.8), (3.10), and (3.4), we have

$$
\begin{aligned}
\left|F_{n_{\nu}}\left(\mu_{j}^{(\nu)}\right)\right| & =\frac{\left|f\left(\lambda_{m_{\nu}+i}\right)\right|}{\left|f\left(\xi_{n_{\nu}}\right)\right|}=\frac{\left|f\left(\lambda_{m_{\nu}+j}\right)\right|}{q\left(\xi_{n_{\nu}}\right)} e^{-C\left(\xi_{n_{\nu}}\right)} \\
& =\frac{\left|f\left(\lambda_{m_{\nu}+j}\right)\right|}{q\left(m_{\nu}+j\right)} \frac{q\left(m_{\nu}+j\right)}{q\left(\xi_{n_{\nu}}\right)} e^{-C\left(\xi_{n_{\nu}}\right) .}
\end{aligned}
$$

But $\left|\xi_{n_{\nu}}-\left(m_{\nu}+j\right)\right| \leqq j+1$, and hence $q\left(m_{\nu}+j\right) / q\left(\xi_{n_{\nu}}\right) \rightarrow 1 \quad(\nu \rightarrow \infty)$; also $C(x)$ $\uparrow \infty$, and $\left|f\left(\lambda_{m_{\nu}+j}\right)\right| \leqq q_{m_{\nu}+j}=q\left(m_{\nu}+j\right)$, so that (3.19) yields, in particular,

$$
G\left(\mu_{j}\right)=\lim _{i=\infty} F_{\nu_{i}}\left(\mu_{j}^{\left(\nu_{i}\right)}\right)=0 \quad(j=0, \pm 1, \cdots) .
$$

Or, it is evident that the sequence $\left\{\mu_{j}\right\}$ satisfies (3.17) for $j=0, \pm 1, \cdots$; from which our assertion (3.15) follows immediately.

Finally, let us apply Jensen's formula:

$$
\int_{0}^{R} \frac{n(r)}{r} d r=\frac{1}{2 \pi} \int_{0}^{2 \pi} \log \left|G\left(R e^{i \theta}\right)\right| d \theta-\log |G(0)|
$$

to our function $G(z)$. Making use of inequalities (3.14) and (3.15), and remembering that $G(0)=1$, we get 


$$
2 R-O(\log R) \leqq \frac{k}{2 \pi} \int_{0}^{2 \pi} R|\sin \theta| d \theta=\frac{2 k R}{\pi}
$$

Dividing by $R$ and sending $R$ to infinity, we obtain $k \geqq \pi$, which, however, contradicts our hypothesis and completes the proof of the theorem.

Theorem 3 a can still be generalized in several directions. Thus, the condition (3.3) was imposed on the sequence $\left\{\lambda_{n}\right\}$ because of its simplicity. The same method yields the following more general theorem.

THEOREM 3b. Let $f(z)$ be an analytic function of exponential type in an angle $|\arg z| \leqq \alpha<\pi / 2$, satisfying (3.2) with $k \geqq 0$ (but not necessarily $k<\pi$ ). Let $\left\{\lambda_{n}\right\}$ be a sequence of complex numbers contained in the strip $x \geqq 0,|y| \leqq M$, and such that $\left|\lambda_{n}-\lambda_{m}\right| \geqq h>0(n \neq m)$. Denote by $N(\xi, r)$ the number of $\lambda_{n}$ 's in the rectangle $|x-\xi| \leqq r,|y| \leqq M$, and put

$$
D=\liminf _{R=\infty} \liminf _{\xi=\infty}\left(\frac{N(\xi, R)}{R}\right) .
$$

Suppose that $D>k / \pi$ and let $\left\{q_{n}\right\}$ be a sequence of positive numbers satisfying (i) and (ii) of Theorem $3 \mathrm{a}$, and such that

$$
q_{n} \geqq \max \left|f\left(\lambda_{m}\right)\right| \quad \text { for } \lambda_{m} \text { in the rectangle } n \leqq x<n+1,|y| \leqq M
$$

(the right side is understood to be zero if there are no $\lambda_{m}$ 's in the rectangle). Then, the conclusion of Theorem 3a holds.

Another generalization consists in considering "multiple" points: that is, when not only $f\left(\lambda_{n}\right)$ is known but also some of its derivatives $f^{\prime}\left(\lambda_{n}\right), \cdots$, $f^{\left(\nu_{n}\right)}\left(\lambda_{n}\right)$. How the theorem must be announced in this case is obvious from our method of proof. Thus, to take a special case, if, in Theorem $3 \mathrm{a}, q_{n}$ $\geqq \max \left(\left|f\left(\lambda_{n}\right)\right|,\left|f^{\prime}\left(\lambda_{n}\right)\right|\right)$ and if $k<2 \pi$ (instead of $k<\pi$ in Theorem 3a), then the conclusion (3.5) holds.

We would also like to note that Theorem $3 \mathrm{a}$ is essentially equivalent to a theorem which was fundamental in our thesis [1, Theorem III] and proved fruitful in investigations of singularities of a class of Dirichlet series. Here we shall show how Theorem 3a can be applied to problems of singularities of Taylor series. (However, the fundamental theorem of [1] is the more "natural" and can easily be generalized to apply to various series having a "power series type" of convergence.) In the following applications only the special case $\lambda_{n}=n$ of Theorem 3 a will be used.

4. Some general remarks and a general gap theorem. Let $\left\{a_{n}\right\}$ be a sequence of positive numbers such that $\lim \sup \left(\left(\log a_{n}\right) / n\right)=0$. We have shown in $[1$, Lemma II $]\left({ }^{5}\right)$ that there always exists a majorant sequence

$\left.{ }^{5}\right)$ We use this opportunity to correct the following printing mistake in [1] where at the top of p. 271 is written: " $\log q_{n} / \Gamma\left(\lambda_{n}+1\right)$ est une fonction concave" and so forth, and it should be: $\log \left(q_{n} / \Gamma\left(\lambda_{n}+1\right)\right)$ and so forth. 
$\left\{q_{n}\right\}$ with the following properties:

(i) $\lim _{n=\infty}\left(q_{n} / q_{n+1}\right)=1$.

(ii) $\lim \sup _{n=\infty} n\left(\log q_{n+1}+\log q_{n-1}-2 \log q_{n}\right) \leqq 1$.

(iii) $q_{n} \geqq a_{n}(n=1,2, \cdots)$ while $q_{n_{k}}=a_{n_{k}}$ for an infinite subsequence $\left\{n_{k}\right\}$ $(k=1,2, \cdots)$.

(Thus, if the sequence $\left\{a_{n}\right\}$ is not bounded, we can take $\log q_{n}$ to be the smallest concave majorant of $\log a_{n}$.) In particular, the above mentioned sequence $\left\{q_{n}\right\}$ satisfies conditions (i) and (ii) of Theorem 3a. Taking $a_{n}=\left|f\left(\lambda_{n}\right)\right|$, we obtain:

Corollary 4a. If, in Theorem 3a, lim sup $\left(\log \left|f\left(\lambda_{n}\right)\right| /\left|\lambda_{n}\right|\right)=0$, then there exists a touching majorant $\left\{q_{n}\right\}$ satisfying (1.6).

Let now $\phi(z)$ be a Taylor series having the unit circle as its circle of convergence

$$
\varphi(z)=\sum_{n=0}^{\infty} a_{n} z^{n} \quad\left(\lim \sup \left|a_{n}\right|^{1 / n}=1\right) .
$$

We lay down the following definition.

Definition 4. A subsequence of coefficients $\left\{a_{n_{k}}\right\}$ of the Taylor series (4.1) will be said to be a sequence of principal coefficients, and the corresponding sequence $\left\{n_{k}\right\}$ a sequence of principal indices, if there exists a positive constant $K$ and a sequence of positive numbers $\left\{q_{n}\right\}$ having properties (i) and (ii) of Theorem 3a and such that

$$
q_{n} \geqq\left|a_{n}\right| \quad(n=0,1, \cdots),
$$

while $q_{n_{k}} \leqq K\left|a_{n_{k}}\right|$ for the given subsequence of coefficients.

From our first remark in the beginning of this section follows: cients.

Corollary 4b. Every Taylor series (4.1) has a sequence of principal coeffi-

Now, it is well known (Carlson [9], see also V. Bernstein [5] where a more general theorem is proved for Dirichlet series) that a necessary and sufficient condition for the function $\phi(z)$, defined by (4.1), to be regular on the symmetric arc of the unit circle $k<|\arg z| \leqq \pi(0<k<\pi)$ is that there will exist a function $f(z)$, analytic and of exponential type in a certain angle $|\arg z| \leqq \alpha$ where it satisfies (3.2), and furthermore such that $f(n)=a_{n}$ $(n=0,1, \cdots)$. In what follows we shall call this function the interpolation function.

We shall apply Theorem $3 a$ to the interpolation function $f(z)$ (with $\left.\lambda_{n}=n\right)$. Let $\left\{n_{k}\right\}(k=1,2, \cdots)$ be a sequence of principal indices of $\phi(z)$, and consider the family of functions $\left\{F_{n_{k}}(z)\right\}$ defined by $F_{n_{k}}(z)=f\left(n_{k}+z\right) / a_{n_{k}}$. It follows from Theorem $3 a$ and Definition 4 that the family is analytic and bounded in every circle $|z| \leqq R$ (for $k \geqq k_{0}(R)$ ), and hence is normal. Further- 
more, by the same argument as is employed in the proof of Theorem 3a, it follows that each limit function $G(z)$ of the family $G(z)=\lim _{k=\infty} F_{n^{\prime} k_{k}}(z)$ is an entire function satisfying

$$
|G(x+i y)| \leqq A e^{k|y|} \quad(k<\pi),
$$

$A$ being a constant independent of the special limit function, and

$$
G(0)=1 .
$$

We also know that the values of $G(z)$ at the integer points are given by

$$
G(m)=\lim _{k=\infty}\left(a_{n^{\prime}{ }_{k}+m} / a_{n^{\prime}{ }_{k}}\right) \quad(m=0, \pm 1, \cdots) \text {. }
$$

Now, given a Taylor series (4.1), let $\left\{n_{k}\right\}$ be a sequence of principal indices, and suppose that there exists a subsequence $\left\{n_{k}^{\prime}\right\}$ of $\left\{n_{k}\right\}$ such that $\lim _{k=\infty}\left(a_{n^{\prime}{ }_{k}+m} / a_{n^{\prime}{ }_{k}}\right)=b_{m}(m=0, \pm 1, \cdots)$ and such that no entire function satisfying (4.3) and $\left(4.3^{\prime}\right)$ can take the values $b_{m}$ at the integer points. Then, it may be concluded from the above remarks that $z=-1$ is a singular point for the series in (4.1). Thus, for example, by a theorem of Cartwright [11, Theorem I], an entire function $G(z)$ satisfying (4.3), and such that $|G(m)|$ $\leqq \exp [-\theta(m)](m=1,2, \cdots)$ where $\theta(m)$ is an increasing sequence satisfying $\sum\left(\theta(m) / m^{2}\right)=\infty$, is identically zero (so that $\left(4.3^{\prime}\right)$ cannot hold). We therefore deduce the following general gap theorem.

THEOREM 4. Let $\phi(z)$ be a Taylor series (4.1), and let $\left\{n_{k}\right\}$ be a sequence of principal indices such that $\lim _{k=\infty}\left(n_{k+1}-n_{k}\right)=\infty$. Suppose that one of the following two conditions is satisfied:

$$
\underset{k=\infty}{\limsup }\left|\frac{a_{n_{k}+m}}{a_{n_{k}}}\right| \leqq e^{-\theta(m)}, \text { or } \quad \limsup _{k=\infty}\left|\frac{a_{n_{k}-m}}{a_{n_{k}}}\right| \leqq e^{-\theta(m)},
$$

for $m=1,2, \cdots$, where $\theta(m)$ is an increasing sequence such that $\sum\left(\theta(m) / m^{2}\right)$ $=\infty$. Then the unit circle is a natural boundary.

This theorem was proved by us (for a class of Dirichlet series) in [1] by another method. (Also in [3] where the present proof is sketched.)

It is seen that no real gaps are required in the gap theorem. It is enough that the coefficients immediately after (or before) the principal coefficients will be relatively small in order that the conclusion will hold. Theorem 4 contains also as a special case the gap theorem of Fabry [14] asserting that the unit circle is a natural boundary for the Taylor series (4.1) if $a_{n}=0$ except for $n=n_{k}$ where $n_{k+1}-n_{k} \rightarrow \infty$. Indeed, in this case it is obvious that any sequence of principal coefficients is to be found among the nonzero coefficients and for any such sequence both conditions (4.4) are satisfied with $\theta(m) \equiv \infty$.

We remark that a very special case of our theorem was proved by Ilieff [15]; namely, the case of the coefficients being bounded while there exists a 
sequence of principal coefficients bounded away from zero and satisfying the first condition (4.4) with $\theta(m) \equiv \infty$. Ilieff's result was generalized by Boas [7] (at about the same time our theorem was announced); but his results are less general and are contained essentially in Theorem 4. As a matter of fact his method applies to cases which can be reduced to Ilieff's theorem by using the Hadamard multiplication theorem. This requires, however, a very regular growth of the principal coefficients. (See also our second remark at the end of the introduction, which deals with the same point in an equivalent form.) Finally we remark that gap theorems with the same general idea were given even earlier by Lösch [20] and Claus [12]. However, their results are different, and in particular they assume that the length of the gaps depends on the magnitude of the coefficients.

5. Generalization of a theorem of Szegö. The following is a well known theorem proved by Szegö [25]:

TheOREM. Let $\phi(z)$ be a Taylor series (4.1) such that its coefficients take only a finite number of different values. Then, a necessary condition that $\phi(z)$ can be continued analytically beyond the unit circle is that the sequence of coefficients be ultimately periodic: $a_{n+l}=a_{n}$ for $n \geqq n_{0}$ and some positive integer $l$.

(It is obvious that the condition is also sufficient and that in this case $\phi(z)$ is a rational function of the form $P(z) /\left(1-z^{l}\right)$.)

We shall prove the following generalization:

THEOREM 5. Let $\phi(z)=\sum_{n=0}^{\infty} a_{n} q_{n} \eta_{n} z^{n}$ where the sequence $\left\{a_{n}\right\}$ takes only a finite number of different values, where $\left\{q_{n}\right\}$ is a sequence such that $q_{n} / q_{n+1} \rightarrow 1$ and $\left(\log q_{n+1}+\log q_{n-1}-2 \log q_{n}\right) \leqq B / n(n=1,2, \cdots$, and $B$ is a constant $)$, and where $\left\{\eta_{n}\right\}$ is a sequence satisfying $\eta_{n} / \eta_{n+1} \rightarrow 1$ and $0<C_{1} \leqq\left|\eta_{n}\right| \leqq C_{2}$ (the C's being constants). Then, a necessary condition that $\phi(z)$ can be continued analytically beyond the unit circle is that the sequence $\left\{a_{n}\right\}$ be ultimately periodic $\left({ }^{6}\right)$.

This theorem was announced by us in [4] and was proved by another method in an unpublished part of our thesis (as a matter of fact we proved there a more general theorem concerning Dirichlet series $g(s)=\sum a_{n} q_{n} \eta_{n} e^{-\lambda_{n} s}$ where $\lambda_{n+1}-\lambda_{n}$ has only a finite number of positive limit points). We remark also that a special case of Theorem 5 (when $q_{n}=n^{\alpha}$ ) was proved independently by Ilieff [16] (see also the same author [17], which is, however, included in the more general theorem for Dirichlet series mentioned above). Another kind of generalization was given by Duffin and Schaeffer [13] who proved that under the conditions of Szegö's theorem, the Taylor series cannot even be bounded in any sector of the unit circle, unless the coefficients are ultimately periodic. The method of proof we shall employ here is similar to

(6) A more precise result could be proved. Namely, that each singular point on the unit circle of $\sum a_{n} z^{n}$ is also a singular point of $\phi(z)$. 
that used by Duffin and Schaeffer in proving their generalization. We shall make use, however, of our more general theorem, Theorem 3a.

Proof of Theorem 5. Suppose that the unit circle is not a natural boundary. There is no loss in generality in assuming $z=-1$ to be regular. (Indeed, if not, we shall consider $\phi_{1}(z)=\phi\left(e^{2 \pi i p / q_{z}}\right)$ where $p$ and $q$ are integers chosen so that $\phi_{1}(z)$ will be regular at $z=-1$. The coefficients of $\phi_{1}(z)$ will take only a finite number of different values and will be periodic (nonperiodic) if the coefficients of $\phi(z)$ are periodic (nonperiodic).) Let $f(z)$ be the interpolation function, associated with the Taylor series, introduced in $\$ 4(f(z)$ is of exponential type in a certain angle, satisfying there (3.2) and is such that $f(n)$ $\left.=a_{n} q_{n} \eta_{n}\right)$. It follows from Theorem 3 a that the family $\left\{F_{n}(z)\right\}$ defined by $F_{n}(z)=f(n+z) / q_{n} \eta_{n}$ is normal in any finite region. Furthermore, by essentially the argument employed in $\$ 4$, it follows that each limit function $G(z)$ is an entire function satisfying (4.3), and such that

$$
\begin{aligned}
G(m)=\lim _{k=\infty} F_{n_{k}}(m)=\lim _{k=\infty} a_{n_{k}+m} \frac{q_{n_{k}+m} \eta_{n_{k}+m}}{q_{n_{k}} \eta_{n_{k}}}=\lim _{k=\infty} a_{n_{k}+m} & \\
& (m=0, \pm 1, \cdots) .
\end{aligned}
$$

Now, suppose, by way of contradiction, that the sequence $\left\{a_{n}\right\}$ is not ultimately periodic. As the sequence takes only a finite number of different values: $b_{1}, b_{2}, \cdots, b_{s} ;\left|b_{i}-b_{j}\right| \geqq b>0(i \neq j)$, it follows in an easy way that for any integer $r=1,2, \cdots$, we can find a pair of integers $p=p(r)$ and $q=q(r), r<p<q$, such that

$$
a_{p} \neq a_{q} \text { while } \quad a_{p-i}=a_{q-i} \quad \text { for } i=1,2, \cdots, r \text {. }
$$

We shall choose two limit functions from the normal family $\left\{F_{n}(z)\right\}: G_{1}(z)$ $=\lim _{i=\infty} F_{p\left(r_{i}\right)}(z)$, where $\left\{p\left(r_{i}\right)\right\}$ is a subsequence of $\{p(r)\}$, and $G_{2}(z)$ $=\lim _{i=\infty} F_{q\left(r_{i}^{\prime}\right)}(z)$, where $\left\{r_{i}^{\prime}\right\}$ is a subsequence of $\left\{r_{i}\right\}$. Let us put $H(z)$ $=G_{1}(z)-G_{2}(z)$. It follows from $\$ 4,(5.1),(5.2)$, and the definition of $H(z)$, that $H(z)$ is an entire function which satisfies

(i) $|H(x+i y)| \leqq 2 A e^{k|y|}(k<\pi)$,

(ii) $H(-m)=0(m=1,2, \cdots)$, and

(iii) $|H(0)| \geqq b>0$.

But, by a theorem of Carlson [8], a function satisfying (i) and (ii) is identically zero. This contradicts (iii) and completes the proof of the theorem.

6. Further applications. A well known theorem by Fabry [14] states that if the Taylor series (4.1) is such that $\lim \left(a_{n} / a_{n+1}\right)=1$, then $z=1$ is singular. We proved in $[1$, Theorem XI] a general theorem from which it follows that if

$$
\lim _{k=\infty} \frac{a_{n_{k}+m}}{a_{n_{k}}}=1 \quad\left(\text { or } \lim _{k=\infty} \frac{a_{n_{k}-m}}{a_{n_{k}}}=1\right)
$$


for $m=1,2, \cdots$, where $\left\{n_{k}\right\}$ is a sequence of principal indices, then $z=1$ is singular.

Now, it is well known that the converse of Fabry's theorem is not true, even when $z=1$ is the only singularity on the unit circle, and even when the singularity is of a relatively simple type. We shall show, however, that (when $z=1$ is the only singularity on the unit circle) the converse of our theorem stated above holds. This will show, in particular, that for any Taylor series (4.1) with a single singularity $z=1$ on the unit circle, $a_{n^{\prime}} / a_{n^{\prime}+1} \rightarrow 1$ when $\left\{n^{\prime}\right\}$ runs through certain blocks of integers, the length of which tends to infinity.

It should be pointed out that much stronger results are known in some special cases. Thus, Jungen [18] in the case where $z=1$ is algebrico-logarithmic, and Whittaker and Wilson [26] in the case where $z=1$ is an essential singularity of finite exponential order, showed that $a_{n^{\prime}} / a_{n^{\prime}+1} \rightarrow 1$ where $\left\{n^{\prime}\right\}$ excludes only a sequence of integers of zero density. The interest of our theorem lies, however, in its generality, as no further assumptions are made upon the nature of the singularity $z=1$. We shall prove:

THEOREM 6. Let $\phi(z)$ be a Taylor series (4.1) with a single singular point, $z=1$, on the unit circle. Let $\left\{n_{k}\right\}$ be an infinite sequence of principal indices. Then,

$$
\lim _{\boldsymbol{k}=\infty} \frac{a_{n_{k}+m}}{a_{n_{k}}}=1 \quad(m= \pm 1, \pm 2, \cdots) .
$$

Proof. Let $f(z)$ be the associated interpolation function introduced in $\S 4$ (thus, $f(z)$ is analytic and of exponential type in a certain angle, satisfying (3.2), and $\left.f(n)=a_{n}(n=0,1, \cdots)\right)$. The point $z=1$ being the only singularity on the unit circle, it can easily be shown that (3.2) can be replaced here by the sharper inequality

$$
\limsup _{r=\infty} \frac{\log \left|f\left(r e^{i \theta}\right)\right|}{r} \leqq \delta(|\theta|)|\sin \theta| \quad(|\theta| \leqq \alpha),
$$

where $\delta(\theta) \quad(0 \leqq \theta \leqq \alpha)$ is continuous, increasing, $\delta(0)=0$, and $\delta(\alpha)<\pi$. Put $F_{n_{k}}(z)=f\left(n_{k}+z\right) / a_{n_{k}}$. By Theorem 3a the family $\left\{F_{n_{k}}(z)\right\}$ is normal in any finite region. We shall show that

$$
\lim _{k=\infty} F_{n_{k}}(z)=1 \quad \text { (uniformly in any bounded region). }
$$

This, of course, will prove the theorem, as we shall get, in particular,

$$
1=\lim _{k=\infty} F_{n_{k}}(m)=\lim _{k=\infty} \frac{f\left(n_{k}+m\right)}{a_{n_{k}}}=\lim _{k=\infty} \frac{a_{n_{k}+m}}{a_{n_{k}}} \quad(m= \pm 1, \pm 2, \cdots),
$$

which is (6.1). 
Now, in order to prove (6.3), it is enough to show that all the limit functions of the family are equal to 1 . Let $G(z)$ be any such limit function. By $\$ 4$ it is an entire function satisfying $G(0)=1$, and

$$
|G(x+i y)| \leqq A e^{\delta(\alpha)|y|}
$$

Or, we could have started by considering $f(z)$ in any smaller angle: $|\arg z|$ $\leqq \alpha^{\prime}<\alpha$. Thus, $\alpha$ in (6.4) can be taken positive but as small as we please. This, combined with $\delta(\theta) \downarrow 0$, yields

(i) $G(z)=O\left(e^{\epsilon|z|}\right)(|z| \rightarrow \infty)$ for any $\epsilon>0$, and

(ii) $G(z)$ is bounded on the entire real axis.

But, it is well known (see, for example, [23, p. 43]) that an entire function satisfying (i) and (ii) is a constant, so that $G(z) \equiv G(0)=1$. This establishes (6.3) and completes the proof of the theorem $\left({ }^{7}\right)$. As a corollary, we obtain $t^{\text {he following. }}$

COROLLARY. Under the conditions of Theorem 6, suppose that there exists a sequence of principal indices $\left\{n_{k}\right\}$ such that $n_{k+1}-n_{k}=O(1)$; then we have $\lim _{n=\infty}\left(a_{n} / a_{n+1}\right)=1$.

\section{BIBLIOGRAPHY}

1. S. Agmon, Sur les séries de Dirichlet, Ann. Ecole Norm. vol. 66 (1949) pp. 263-310.

2. - Fonctions analytiques dans un angle et propriêtés des séries de Taylor, C. R. Acad. Sci. Paris vol. 226 (1948) pp. 1497-1499. 1674.

3. - Sur deux théorèmes de Fabry, C. R. Acad. Sci. Paris vol. 226 (1948) pp. 1673-

4. - Sur le comportement d'une série de Taylor sur le cercle de convergence, C. R. Acad. Sci. Paris vol. 226 (1948) pp. 1875-1876.

5. V. Bernstein, Leçons sur les progrès récents de la théorie des séries de Dirichlet, Paris, Gauthier-Villars, 1933. 169.

6. R. P. Boas, Jr., Entire functions bounded on a line, Duke Math. J. vol. 6 (1940) pp. 148-

7. - A class of gap theorems, Duke Math. J. vol. 15 (1948) pp. 725-728.

8. F. Carlson, Sur une classe de sêries de Taylor, Upsala thesis (1914).

9. - Über Potenzreihen mit endlich vielen verschiedenen Koeffizienten, Math. Ann. vol. 79 (1919) pp. 237-245.

10. M. L. Cartwright, On certain integral functions of order one, Quart. J. Math. Oxford ser. vol. 7 (1936) pp. 46-55.

11. - Some uniqueness theorems, Proc. London Math. Soc. vol. 41 (1936) pp. 33-47.

12. H. Claus, Neue Bedingungen für die Nichtfortsetzbarkeit von Potenzreihen, Math. Zeit. vol. 49 (1943) pp. 161-191.

13. R. J. Duffin and A. C. Schaeffer, Power series with bounded coefficients, Amer. J. Math. vol. 67 (1945) pp. 141-154.

14. E. Fabry, Sur les points singuliers d'une fonction donnée par son développement en série et sur l'impossibilité du prolongement analytique dans les cas très généraux, Ann. École Norm. vol. 13 (1896) pp. 367-399.

(7) It can be shown that the constant $A$ in (6.4) could be taken to be independent of $\alpha$, which will simplify the last part of the proof. 
15. L. Ilieff, Analytisch nichtfortsetzbare Potenzreihen, Annuaire de l'Université de Sofia, Faculté Physico-Mathematique, part 1, vol. 42 (1946) pp. 67-81.

16. - Analytisch nichtfortsetzbare Potenzreihen, Comptes Rendus de l'Académie Bulgare des Sciences vol. 1, no. 1 (1948) pp. 25-28.

17. — Über die Verteilung der singulären Stellen einer Klasse Dirichletscher Reihen in der Umgebung der Konvergenzgeraden, Comptes Rendus de l'Académie Bulgare des Sciences vol. 1 , nos. 2 and 3 (1948) pp. 19-22.

18. R. Jungen, Sur les séries de Taylor n'ayant que des singularités algébrico-logarithmiques sur leur cercle de convergence, Comment. Math. Helv. vol. 3 (1931) pp. 266-306.

19. N. Levinson, Gap and density theorems, Amer. Math. Soc. Colloquium Publications, vol. $26,1940$.

20. F. Lösch, Über nichtfortsetzbare Potenzreihen mit Lücken, Math. Zeit. vol. 32 (1930) pp. $415-421$.

21. A. J. Macintyre, Laplace's transformation and integral functions, Proc. London Math. Soc. (2) vol. 45 (1938-1939) pp. 1-20.

22. F. and R. Nevanlinna, Über die eigenschaften einer analytischer Funktion in der Umgebung einer singularen Stelle oder Linie, Acta Societatis Scientiarum Fennicae vol. 50, no. 5 (1922).

23. R. Nevanlinna, Eindeutige analytische Funktionen, Berlin, 1936.

24. A. Pfluger, Über das Anwachsen von Funktionen, die in einem Winkelraum regulär und von Exponentialtypus sind, Composito Math. vol. 4 (1937) pp. 367-372.

25. G. Szegö, Tschebyscheffsche Polynome und nichtfortsetzbare Potenzreihen, Math. Ann. vol. 87 (1922) pp. 90-111.

26. J. M. Whittaker and R. Wilson, Fabry's theorem and the isolated essential singularity of finite exponential order, J. London Math. Soc. vol. 14 (1939) pp. 202-208.

The Rice Institute, Houston, Tex. 